\title{
Promoting Physical Activity In Adolescent Cancer Survivors
}

\author{
Amanda Wurz ${ }^{1}$, Jennifer Brunet, $\mathrm{PhD}^{1}$ \\ ${ }^{1}$ School of Human Kinetics, University of Ottawa
}

A BSTRACT

Self-management strategies, such as physical activity, have been identified to help young cancer survivors reduce or control the side effects that accompany modern cancer therapies whilst improving their overall quality of life. Despite the known benefits of physical activity, the majority of young cancer survivors are not meeting recommended guidelines. In this article, we discuss knowledge translation activities that are taking place across Canada to develop and disseminate resources to healthcare providers in an effort to improve physical activity counselling, and ultimately participation for adolescent cancer survivors.

RÉ S U É

\section{INTRODUCTION}

Progress in cancer treatment protocols offer hope for the 7,500 boys and girls aged 15 to 19 years living in North America who are diagnosed with cancer each year $[1,2]$. Lymphomas, leukemias, germ cell tumours, central nervous system tumours, and melanomas are the most common types of cancers diagnosed in adolescents $[1,2]$. Fortunately, recent survival estimates show that $80 \%$ will live for at least 5 years after their cancer is diagnosed $[3,4]$.

To assist this growing population of adolescent cancer survivors (i.e., adolescents who have been diagnosed with cancer, from the time of diagnosis onward [5]) on their survivorship journey, it is important to recognize the unique challenges they may face. Adolescence is a complex life stage associated with developmental demands that differ from those experienced during childhood and adulthood. Significant physical, psychological, and social challenges and changes characterize this phase in life [6]. As such, a diagnosis of cancer during adolescence may be particularly detrimental as it can interrupt boys' and girls' healthy development and thwart their ability to cope with the disease [7]. Indeed, many adolescent cancer survivors experience a host of adverse physical, psychological, and social side effects as a result

Keywords: Physical activity; Counselling; Promotion; Adolescent oncology of their treatment regimens (which may include a combination of surgery, chemotherapy, radiation, and/or hematopoietic stem cell transplant) [8-10].

In addition, it is estimated that $95 \%$ of young cancer survivors will develop at least one chronic health condition by the age of 45 years, and $80 \%$ will develop a serious, disabling, and/or lifethreatening condition (e.g., pulmonary, cardiac, organ dysfunctions, neurocognitive impairment) as a result of their treatments [11]. Therefore, although they are 'cancer free', this population is far from 'disease free'. As well, young cancer survivors are 8.4 times more likely to die 5 years after their diagnosis compared to their healthy peers due to cancer recurrence and disease progression (57.5\% of deaths), subsequent cancers ( $18.6 \%$ of deaths), and diseases of the circulatory (6.9\% of deaths) and respiratory systems (2.6\% of deaths) [12]. So while preventing cancer in the first place remains a top public health priority, a key challenge is to promote longevity, health, and quality of life amongst those diagnosed. For this reason, several researchers and stakeholders are focusing their efforts on helping young cancer survivors selfmanage the negative side effects that may occur during treatment, immediately after, and/or years into survivorship. These endeavours are necessary to empower adolescent cancer survivors and positively impact their overall health and quality of life [13]. 


\section{PHYSICAL ACTIVITY AS A SELF-CARE STRATEGY}

As researchers, we advocate for the use of physical activity as a self-care strategy to help adolescent cancer survivors address the negative side effects associated with the disease and its treatments, as well as help them enhance their health and quality of life throughout survivorship. Our focus on physical activity is based on the mounting evidence that it can improve physical, psychological, and social health amongst cancer survivors across the lifespan $[14,15]$. It is also based on evidence that physical activity may help reduce the risk of several health conditions, such as cancer recurrence, co-morbidities (e.g., obesity, cardiovascular disease, second cancers, organ dysfunctions, neurocognitive impairment), and premature mortality [15-18].

Despite an abundance of evidence demonstrating the health benefits of physical activity, there are marked declines in physical activity participation amongst adolescents following cancer diagnosis, which generally remain low months, years, or decades into survivorship [19]. In fact, a recent study found that $65 \%$ of child and adolescent cancer survivors living in the United States were not meeting guidelines that recommend at least $60 \mathrm{~min}-$ utes of physical activity daily [20]. As well, a recent review suggests adult survivors of childhood or adolescent cancers were less active than non-cancer controls [21]. These low rates may be due to the additional cancer-related barriers to physical activity experienced by adolescent cancer survivors, such as short- and long-term side effects, physical deconditioning, and overprotective attitudes of caregivers [22, 23]. Despite this, it should be noted that adolescent cancer survivors want information about physical activity [24] and are motivated to make positive lifestyle changes [25]. Therefore, finding ways to promote physical activity in this population is critical to enhance physical, psychological, and social health, and reduce morbidity and mortality.

\section{HEALTHCARE PROVIDERS AS PHYSICAL ACTIVITY ADVOCATES}

Given that adolescent cancer survivors experience close contact with their healthcare team during treatment and into survivorship, healthcare providers are in a key position to influence their patients' lifestyle behaviours through counselling. For instance, by conveying information about the importance of physical activity and prescribing it during clinic visits, adolescent cancer survivors may be more inclined to adopt an active lifestyle. Based on a recent systematic review, physical activity counselling may effectively enhance physical activity participation [26]. Although this conclusion is based on studies conducted with disease-free sedentary youth and adults [26], it points to the potential value of physical activity counselling in the cancer setting. In fact, emerging research performed with adult cancer populations has found that physical activity counselling led by healthcare providers is effective at increasing participation [27]. Considering that as many as $75 \%$ of adolescents agree with the statement "If my doctor told me to exercise I would do so" [28], physical activity counselling is likely to be effective in this population as well. Unfortunately, many adolescent cancer survivors living in North America are not receiving such counselling from their healthcare providers $[24,29]$.

Why is this the case? In adult cancer populations, insufficient resources (e.g., lack of money, time, and space), lack of expertise in the area of physical activity, and limited awareness of benefits of physical activity are key barriers to routine physical activity counselling in clinical practice [30]. In a similar vein, key barriers to physical activity counselling in pediatric cancer populations include a lack of knowledge and resources, as well as a belief that patients do not adhere to physical activity recommendations [31]. As such, equipping healthcare providers with information about the benefits of physical activity, providing them with evidence-based resources, and informing them that adolescents want this information, may increase the frequency of physical activity counselling in practice.

Recent recommendations by the National Comprehensive Cancer Network urge healthcare providers to give physical activity information to adolescent and young adult cancer survivors [32]. In doing so, there are special age-appropriate considerations that should be taken into account. In recognition of adolescents' growing desire to be independent and autonomous, every effort should be made to ensure that physical activity counselling is delivered to adolescent cancer survivors in a manner that is supportive, rather than controlling [33, 34]. Specifically, efforts should be made to counsel adolescent cancer survivors in a respectful, positive, non-judgmental way, and frame it as what they can do, versus what they cannot do [33, 34]. Further, some adolescents may be accompanied by their parents/guardians; in these instances care should be taken to give information directly to adolescents to facilitate feelings of autonomy and control [35].

\section{TRANSLATING THE EVIDENCE: PLANS FOR ACTION AND EXISTING RESOURCES}

To equip healthcare providers with the information they need to provide regular physical activity counselling to their patients, several groups are undertaking knowledge synthesis and resource development projects to translate knowledge into practice [36-39]. For example, we have synthesized the best available evidence from randomized controlled trials and controlled clinical trials exploring the effects of physical activity on health and quality of life outcomes with adolescent cancer survivors. In our review, one randomized controlled trial and two controlled clinical trials met our inclusion criteria. Consequently, we are unable to determine whether physical activity has an effect on health and quality of life for adolescent cancer survivors given the very 
limited data and methodological limitations of the reviewed studies. Despite this, the studies included in our review provided evidence that physical activity is both safe and feasible. These findings are important as they can alleviate any concerns healthcare providers might have about the potential harm of physical activity during and/or after treatment for adolescent cancer survivors. We plan on publishing the results of our review to inform the scientific community of the safety of physical activity, as well as highlight the lack of research in this area. We also plan on presenting the findings to knowledge users (e.g., healthcare providers, adolescents). This work will be a useful starting point for the creation of physical activity information and resources such as pamphlets that will summarize our findings, as well as integrate guidelines and recommendations from other research and resources [36-39]. Lastly, this work will help lay the foundation for future studies and community outreach that aim to develop optimal physical activity programs for this population.

In addition to our own efforts, others have compiled evidence on the safety and benefits of physical activity, and used this evidence to develop critical resources and physical activity guidelines. For example, the Canadian Cancer Society provides a summary of the benefits of physical activity on their website and on print materials [39], and further recommends that cancer survivors follow nationally recognized physical activity guidelines developed by the Canadian Society for Exercise Physiology (CSEP) [37]. These guidelines, which were reviewed by healthcare providers, academics, international content experts, governmental and non-governmental organizations, and community members, encourage adolescent cancer survivors to engage in 60 minutes of physical activity daily [37]. Similarly, the American College of Sports Medicine and a team from the University of Calgary have developed evidence-based guidelines for adults [38] and younger (i.e., children and adolescents) cancer populations [36] respectively. In both cases, the guidelines were developed using rigorous processes that met international standards for guideline development.

Considering the current body of knowledge on the benefits of physical activity for cancer survivors and existing physical activity guidelines and resources, it is clear that adolescent cancer survivors can and should be engaging in physical activity. Special consideration should be taken with survivors on- and offtreatment, including carefully considering contraindications and co-morbidities that could interfere with cancer survivors' ability to perform physical activity (e.g., avascular necrosis, pulmonary disease, neurological problems, general performance limitations) $[36,40]$. Furthermore, healthcare providers should take into account adolescents' past physical activity patterns, current levels of physical fitness, and activity preferences when recommending physical activity. They should also consider referring their patients to physical activity specialists (preferably specialists who have received training in cancer rehabilitation) [36-39]. Moving forward, the goal is to ensure healthcare providers use existing and emergent information and resources as tools for making physical activity recommendations for their patients, and incorporate physical activity counselling into routine preventive and rehabilitative cancer care.

\section{CONCLUSION}

A large and convincing body of evidence shows the benefits of physical activity for cancer survivors across the lifespan. Healthcare providers need to be made aware of this evidence so that they may confidently counsel adolescent cancer survivors to be physically active. It is hoped that the development of resources will provide healthcare providers with the knowledge and tools necessary to promote physical activity participation amongst adolescent cancer survivors, and thus reduce the burden of cancer on an already taxed healthcare system. Encouraging physical activity is the first step in helping the growing population of adolescent cancer survivors experience long-term health and enhanced quality of life.

\section{REFERENCES}

1. American Cancer Society. Cancer facts \& figures 2014. Atlanta, GA: American Cancer Society; 2014. p.25

2. Canadian Cancer Society's Advisory Committee on Cancer Statistics. Canadian Cancer Statistics 2014. Toronto, ON: Canadian Cancer Society; 2014.

3. Smith MA, Seibel NL, Altekruse SF, et al. Outcomes for children and adolescents with cancer: challenges for the twenty-first century. J Clin Oncol. 2010;28(15):2625-34.

4. Ward E, DeSantis C, Robbins A, et al. Childhood and adolescent cancer statistics 2014. CA Cancer J Clin. 2014;64(2):83-103.

5. National Cancer Institute [Internet]: National Cancer Institute dictionary of cancer terms: survivor. [cited 2015 Apr 3]. Available from: http://www. cancer.gov/dictionary.

6. Steinberg L, Morris AS. Adolescent development. Annu Rev Psychol. 2001;52:83-110.

7. Epelman $\mathrm{CL}$. The adolescent and young adult with cancer: state of the art - psychosocial aspects. Curr Oncol Rep. 2013;15(4):325-31.

8. Children's Oncology Group. Long-term follow-up guidelines for survivors of childhood, adolescent and young adult cancers, Version 4.0. Arcadia, CA: Children's Oncology Group; 2013. p. 2

9. Erickson JM, Macpherson CF, Ameringer S, et al. Symptoms and symptom clusters in adolescents receiving cancer treatment: a review of the literature. Int J Nurs Stud. 2013;50(6):847-69.

10. San Juan AF, Wolin K, Lucia A. Physical activity and pediatric cancer survivorship. Recent Results Cancer Res. 2011;186:319-47.

11. Hudson MM, Ness KK, Gurney JG, et al. Clinical ascertainment of health outcomes among adults treated for childhood cancer. JAMA. 2013;309(22):2371-81.

12. Mertens AC, Liu Q, Neglia JP, et al. Cause-specific late mortality among 5-year survivors of childhood cancer: the Childhood Cancer Survivor Study. J Natl Cancer Inst. 2008;100(19):1368-79.

13. McCorkle R, Ercolano E, Lazenby M, et al. Self-management: enabling and empowering patients living with cancer as a chronic illness. CA Cancer J Clin. 2011;61(1):50-62.

14. Baumann FT, Bloch W, Beulertz J. Clinical exercise interventions in pediatric oncology: a systematic review. Pediatr Res. 2013;74(4):366-74.

15. Sabiston CM, Brunet, J. Reviewing the benefits of physical activity during cancer survivorship. Am J Lifestyle Med. 2012;6.

16. Ballard-Barbash R, Friedenreich CM, Courneya KS, et al. Physical activity, biomarkers, and disease outcomes in cancer survivors: a systematic review. J Natl Cancer Inst. 2012;104(11):815-40.

17. Kenfield SA, Stampfer MJ, Giovannucci E, et al. Physical activity and survival after prostate cancer diagnosis in the health professionals follow-up study. J Clin Oncol. 2011;29(6):726-32.

18. White J, Flohr JA, Winter SS, et al. Potential benefits of physical activ- 
ity for children with acute lymphoblastic leukaemia. Pediatr Rehabil. 2005;8(1):53-8.

19. Winter C, Muller C, Hoffmann C, et al. Physical activity and childhood cancer. Pediatr Blood Cancer. 2010;54(4):501-10.

20. Badr H, Chandra J, Paxton RJ, et al. Health-related quality of life, lifestyle behaviors, and intervention preferences of survivors of childhood cancer. J Cancer Surviv. 2013;7(4):523-34.

21. Stolley MR, Restrepo J, Sharp LK. Diet and physical activity in childhood cancer survivors: a review of the literature. Ann Behav Med. 2010;39(3):232-49.

22. Arroyave WD, Clipp EC, Miller PE, et al. Childhood cancer survivors' perceived barriers to improving exercise and dietary behaviors. Oncol Nurs Forum. 2008;35(1):121-30.

23. Gotte $\mathrm{M}$, Kesting $\mathrm{S}$, Winter $\mathrm{C}$, et al. Experience of barriers and motivations for physical activities and exercise during treatment of pediatric patients with cancer. Pediatr Blood Cancer. 2014;61(9):1632-7.

24. Hall AE, Boyes AW, Bowman J, et al. Young adult cancer survivors' psychosocial well-being: a cross-sectional study assessing quality of life, unmet needs, and health behaviors. Support Care Cancer. 2012;20(6):1333-41.

25. Wright M, Bryans A, Gray K, et al. Physical activity in adolescents following treatment for cancer: influencing factors. Leuk Res Treatment. 2013;2013:592395.

26. Orrow G, Kinmonth AL, Sanderson S, et al. Effectiveness of physical activity promotion based in primary care: systematic review and meta-analysis of randomised controlled trials. BMJ. 2012;344:e1389.

27. Jones LW, Courneya KS. Exercise discussions during cancer treatment consultations. Cancer Pract. 2002;10(2):66-74.

28. Desmond SM, Price JH, Lock RS, et al. Urban black and white adolescents' physical fitness status and perceptions of exercise. J Sch Health. 1990;60(5):220-6.

29. Zebrack B. Information and service needs for young adult cancer survivors. Support Care Cancer. 2009;17(4):349-57.

30. Santa Mina D, Alibhai SM, Matthew AG et al. Exercise in clinical cancer care: a call to action and program development description. Curr Oncol. 2012;19(3):e136-44.

31. Keats MR, Culos-Reed SN, Courneya KS. An examination of the beliefs, attitudes and counselling practices of paediatric oncologists toward physical activity: a provincial survey. Paediatr Child Health. 2007;12(4):289-93.

32. Coccia PF, Altman J, Bhatia S, et al. Adolescent and young adult oncology: clinical practice guidelines in oncology. J Natl Compr Canc Netw. 2012;10(9):1112-50.

33. Zebrack B, Chesler MA, Kaplan S. To foster healing among adolescents and young adults with cancer: what helps? what hurts? Support Care Cancer. 2010;18(1):131-5.

34. D’Agostino NM, Penney A, Zebrack B. Providing developmentally appropriate psychosocial care to adolescent and young adult cancer survivors. Cancer. 2011;117(10 Suppl):2329-34.

35. Palmer S, Mitchell A, Thompson K, et al. Unmet needs among adolescent cancer patients: a pilot study. Palliat Support Care. 2007;5(2):127-34.

36. Chamorro-Vina C, Keats, M, Culos-Reed, SN. Pediatric oncology exercise manual, Version 1. Calgary, AB: Faculty of Kinesiology, University of $\mathrm{Cal}-$ gary, The Health and Wellness Lab; 2014.

37. Canadian Society for Exercise Physiology [Internet]: Canadian physical activity guidelines [cited 2015 Mar 5]. Available from: http://www.csep.ca/ english/view. aspx=949.

38. Schmitz KH, Courneya KS, Matthews C, et al. American College of Sports Medicine roundtable on exercise guidelines for cancer survivors. Med Sci Sports Exerc. 2010;42(7):1409-26.

39. Canadian Cancer Society [Internet]: Physical activity. [cited 2015 Mar 5]. Available from: http://www.cancer.ca/en/preven- tion-and-screening/ live-well/nutrition-and-fitness/physical-activity/ region=on.

40. Diller L, Chow EJ, Gurney JG, et al. Chronic disease in the Childhood Cancer Survivor Study cohort: a review of published findings. J Clin Oncol. 2009;27(14):2339-55. 